sequences among mammalian lineages. By contrast, in the pufferfish, single nucleotide differences and small indels are much more common, which probably reflects the adverse effects of large indels in this compact genome.

As the authors indicate, this study points to 'myriad avenues' for future evolutionary and functional studies, which shows why the comparative approach is becoming the core theme of genomics research. However, the success of this targeted approach begs the question of how many more vertebrate genomes we need to sequence completely?

Nick Campbell

20) References and links ORIGINAL RESEARCH PAPER

Thomas, J. W. et al. Comparative analyses of multispecies sequences from targeted genomic species sequences from targeted geno
regions. Nature 424, 788-793 (2003) regions. Nature 424, 788-793 (2003)
FURTHER READING Ureta-Vidal, A. et al. Comparative genomics: genome-wide analysis in metazoan eukaryotes. Nature Rev. Genet.4. 251-262 (2003)

\section{WEB SITE}

NIH Intramural Sequencing Centre:

http://www.nisc.nih.gov

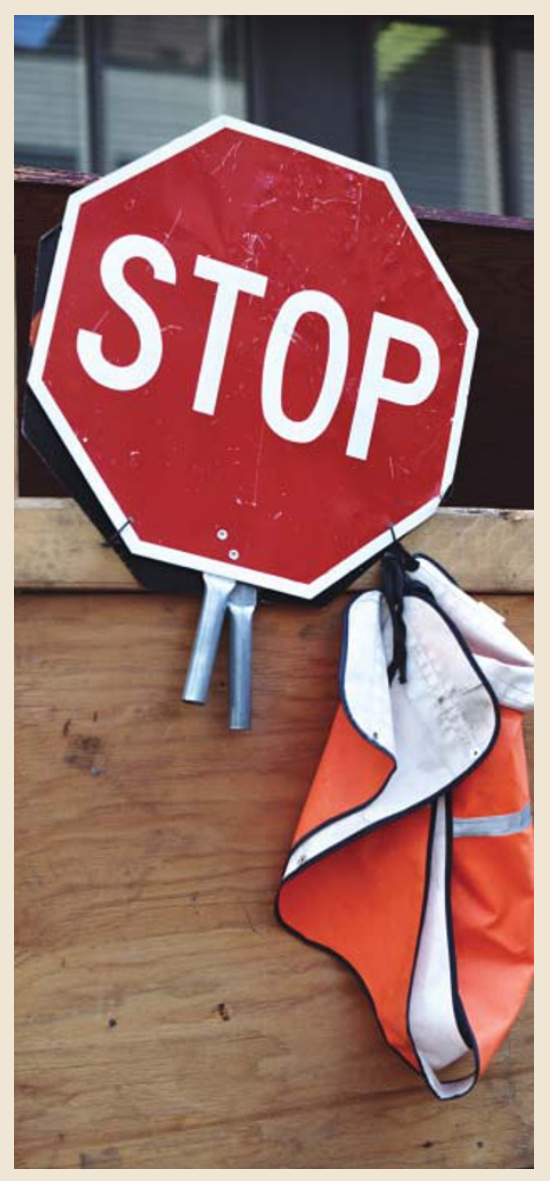

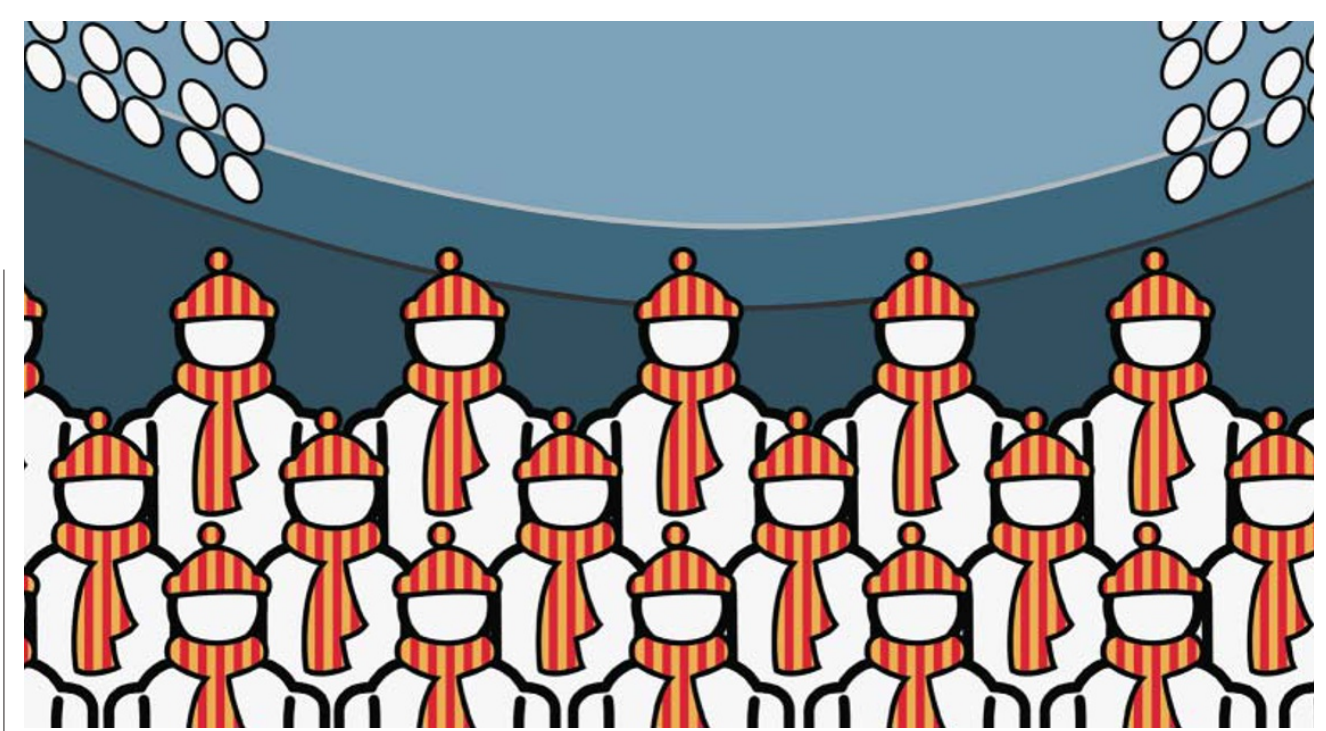

GENE EXPRESSION

\section{Repeat performance silences the crowd}

A newly discovered role in epigenetic gene silencing for the retrotransposon long terminal repeats (LTRs) that are scattered throughout eukaryotic genomes indicates that they might be involved in gene regulation during development.

We have known for some time that repetitive DNA and gene silencing are linked, and the idea that repeats might be involved in gene regulation is an old one. However, direct evidence was lacking until now.

The initial aim of Vera Schramke and Robin Allshire's study was to determine whether RNA interference (RNAi) - through both posttranscriptional gene silencing (PTGS) and chromatin-based gene silencing (CBGS) - could silence non-centromeric genes in fission yeast in the same way that it had been shown to silence centromeric repeats.

Using RNAi, the authors were able to silence the ura 4+ gene both at its native centromeric locus and when inserted at other non-centromeric locations, by expressing an inverted 280-bp section of the gene - short hairpin (shuraSE) in the same strain. As expected, silencing was abolished in strains in which genes that encode crucial components of the PTGS pathway, such as dicer (dcr1), were mutated. Less predictably, ura $^{+}$ silencing was also abolished in a strain that lacked the histone $\mathrm{H} 3$ lysine 9 methyltransferase Clr4, which was previously only known to be involved in the CBGS pathway.

By contrast, cells that lacked another CBGS component, the HP1 orthologue Swi6, retained silencing. So, it seems that Clr4 is not only involved in silencing through chromatin modification, but is also a component of the RNAi complex that generates the short interfering RNAs (siRNAs) that are the effectors of PTGS.

Schramke and Allshire, using chromatin immunoprecipitation, also assessed whether the shuraSE-silenced non-centromeric $\mathrm{ura}^{+}$gene had the chromatin modifications - methylated histone $\mathrm{H} 3$ and bound Swi6 - that are characteristic of silenced centromeric repeats. Sure enough, not only did the relocated rra $^{+}$ have an identical pattern of chromatin modification to centromeric repeats, but experiments in a strain that lacked Swi6 function indicated that, like these repeats, Swi6 was needed to spread silencing from the nucleation site and Rad21-cohesin was also recruited just like at centromeres.

To test whether such RNAi-mediated chromatin modifications are involved in endogenous gene regulation, the authors used RT-PCR to assess whether the silencing of meiotically-induced genes could be abolished in strains that lacked key components of the RNAi pathway, including Clr4 and Swi6. A notable pattern emerged: only genes with promoters close to an LTR were de-repressed (that is, unsilenced) in cells that lacked these RNAi-pathway components.

Further experiments showed that - at least for D11.02 and meu6 - RNAi-dependent modifications at LTRs mediate transcriptional repression, and removal of these LTRs leads to constitutive expression (de-repression) of the adjacent genes. Taken together, these results indicate that LTRs are needed to silence meiotically induced genes during vegetative growth. More generally, the authors conclude that LTRs can act as developmental effectors through the RNAi pathway, restricting the expression of a gene to a distinct differentiation pathway.

These fascinating results in fission yeast show that despite the recent flurry of RNAi research activity in a plethora of models, we still have a way to go before we fully understand this process. After Schramke and Allshire's study, the role of repetitive DNA elements in RNAi in other models is likely to be the focus of many new studies.

(2) References and links

Nick Campbell

ORIGINAL RESEARCH PAPER Schramke, V. \& Allshire, R. Hairpin RNAs and retrotransposon LTRs effect RNAi and chromatin-based gene silencing. Science 17 July 2003 (10.1126/science.1086870) FURTHER READING Hannon, G. J. RNA interference. Nature $\mathbf{4 1 8}$ 244-251 (2002)

WEB SITE

Robin Allshire's laboratory: http://www.wcb.ed.ac.uk/allshire.htm 